www.nature.com/pj

\title{
Structure of nylon 6/iodine complex. 5: variation of intercalation in complexes induced by humidification
}

\begin{abstract}
Akio Kawaguchi ${ }^{1}$ and Naoki Tsurutani ${ }^{2}$
A nylon 6/iodine complex prepared with nylon 6 (polyamide-6, PA6) and provided by immersion in aqueous solutions of polyiodide, $\mathrm{I}_{n}^{-}$, showed intercalated structures composed of iodine in which polyiodide ions are coordinated between hydrogen-bonded sheets of nylon 6 . This work reports that these intercalated structures can be modified by aging under their swollen condition. After iodine doping with an $\mathrm{I}_{2}-\mathrm{KI}$ aqueous solution, the most significant intercalation shows primarily 1.56-nm spacing in its wide-angle $X$-ray diffraction pattern, which is considered to be due to the intercalation of iodine layers between every hydrogen-bonded sheet of nylon 6 . Although this spacing is almost stable under dry conditions, other structures showing 1.95-nm spacing or greater were revealed or vanished during aging under moist conditions. It is remarkable that the coordinated structures composed of ordered crystalline regions of the polymer dynamically and actively respond to moisture; the diffusion of ions or low-molecular-weight molecules into the iodinated polymer can be achieved easily within the crystallites, and such dynamics can be realized with chain- or sheet-like structures in polymeric materials.
\end{abstract}

Polymer Journal (2011) 43, 385-389; doi:10.1038/pj.2011.9; published online 16 February 2011

Keywords: intercalated structure; iodine; moisture; nylon

\section{INTRODUCTION}

Since the first studies on the $\gamma$-phase of nylon 6 (polyamide-6, PA6), iodine doping of nylon 6 has been regarded as an effective procedure for the interconversion of hydrogen-bonded structures formed within crystallites of nylon 6. Iodine doping has been applied to convert the $\alpha$-phase of nylon 6 crystallite to the $\gamma$-phase. The doping process, which involves the immersion of nylon 6 samples in an $\mathrm{I}_{2}-\mathrm{KI}$ aqueous solution, and a following 'de-doping process (reduction)' using an aqueous solution of $\mathrm{Na}_{2} \mathrm{~S}_{2} \mathrm{O}_{3}$, leads to the conversion from the $\alpha$-phase crystallite to the $\gamma$-phase: ' $\alpha-\gamma$ conversion., ${ }^{1,2}$ However, unlike the relatively easily executed experimental procedure, the structure of nylon samples in their iodine-doped stage as an intermediate state has not been precisely investigated, and the mechanism for the $\alpha-\gamma$ conversion has not been satisfactorily clarified. Since the 1980s, some investigations have partially elucidated the characteristic behaviors and structures of iodine-doped nylon 6 (nylon 6/iodine complex), although a new inadequacy has prevented researchers from developing an explicit description. Through certain experiments, some important behaviors have been reported, such as the transitions of ordered structures of adsorbed polyiodide ions $\left(\mathrm{I}_{n}{ }^{-} ; n=3,5\right)$ after doping and the modification of intercalation or orientation induced by the diffusion of ions. ${ }^{3-12}$ The explanation of these behaviors has been hampered because of the following reasons: transparent observation is difficult because of the optical absorption of iodine; the adsorbed polyiodide ions can be transformed after doping; and a depth distribution exists at each stage of aging after doping. However, there exists some generality in treating hydrophilic polymers when we consider some more familiar concepts related to the study of the complex: hydrogen bonding, coordination, ordered states, orientation, diffusion and gelation. Iodine-doped nylon 6 may show unique behaviors, but we may find some generalities applied to other polymers.

One of the characteristic behaviors of the complex versus those of undoped nylon 6 we have researched is its activated ionic mobility through the complex in a humid environment. In previous studies, we observed a quick response in conduction through the complex because of moisture; swelling in the moist environment enhances ionic conduction through the complex. ${ }^{12}$ Conduction in the complex is activated by humidification and it depends on counter ions (cations), which are doped together with the polyiodide ions, and this activation can be observed in a minute or more just after moving the dried iodine-doped complexes to moist environment. Such a quick response induced by moisture in a few minutes is attributed to a particular process in the amorphous region of the complex. Yet investigation of the relative increase in mass $(\Delta m)$, which is defined as increasing against mass of nondoped nylon 6 , of the complex in the moist environment suggested another diffusion process in response to moisture; the slow diffusion, compared with the quick diffusion through the amorphous region, may be attributed to the transfer of dopants to the crystalline region of the complex..$^{13}$ Though diffusion in polymer crystallites is generally neglected, diffusion through the

${ }^{1}$ Division of Quantum Beam Material Science, Research Reactor Institute, Kyoto University, Kumatori-cho, Osaka, Japan and ${ }^{2}$ Department of Physics, Faculty of Science, Kyoto University, Kyoto, Japan

Correspondence: Dr A Kawaguchi, Research Reactor Institute, Kyoto University, Kumatori-cho, Sennan-gun, Osaka 590-0494, Japan.

E-mail: akawagch@rri.kyoto-u.ac.jp

Received 6 September 2010; revised 6 December 2010; accepted 20 December 2010; published online 16 February 2011 
nano-spaces of nylon 6 or reaction of the iodine-doped complex is more active than estimated.

In this paper, the response of a coordinated structure in the ordered region of an iodinated nylon complex is reported for a complex aged in a moist environment. Swelling of the complex by moisture induces modification of the ordered structures composed in the crystallites of nylon 6 .

\section{EXPERIMENTAL PROCEDURE}

\section{Thin-film sample enclosed in vessel maintaining moisture for} wide-angle $\mathrm{X}$-ray diffraction

As thin-film samples, nylon 6 films of Rayfan NO $100 \mu \mathrm{m}$ in thickness (type \#1401) were provided by Toray Plastic Films (Shiga, Japan) and were cut into pieces with dimensions $\sim 5 \times 10 \mathrm{~mm}$. After annealing in vacuum at $200{ }^{\circ} \mathrm{C}$ for 20 days (first drying process), the samples were immersed in aqueous $\mathrm{I}_{2}-\mathrm{KI}$ solution in 3 -ml glass capsules at $5{ }^{\circ} \mathrm{C}$ (iodine-doping process). This polyiodide solution was prepared at a concentration of $0.8 \mathrm{~N}: 0.4 \mathrm{moll}^{-1}$ for $\mathrm{I}_{2}$ and $1.2-1.4 \mathrm{moll}^{-1}$ for KI. After immersing the samples in solution for more than 3 weeks, iodine doping was stopped by rinsing the thin filmy samples in water. The samples were then returned to vacuum and aged for more than 3 weeks to minimize the effects induced by volatile elements at room temperature (second drying process).

The thin filmy samples were swollen in an enclosing vessel used to perform wide-angle X-ray diffraction (WAXD). To maintain a constant relative humidity $(\mathrm{RH})$ during the WAXD measurements, the original vessel was prepared for a humid environment; diffraction could be observed through a window of poly(ethylene terephthalate) (PET) or polyimide film, and moist air washed through pure water was pumped into the vessel. On being set in the vessel, the dried film samples were immediately enclosed within to minimize disturbance by the outer environment. The $\mathrm{RH}$ and temperature in the vessel were monitored by a humidity-temperature logger (Shin-ei, Hyogo, Japan, TRH$3 \mathrm{~A}$ and THP-25A), and these environmental data were recorded using a personnel computer. We then traced the diffraction intensities of the samples within the closed vessel as a function of time under nearly constant RH and temperature.

\section{Swelling plane samples in desiccator}

As a thick sample, nylon 6 pellets (Aldrich Chemical, Milwaukee, WI, USA) were melted on a hot plate at $230^{\circ} \mathrm{C}$ and re-crystallized into plane samples $0.5-$ $1.0 \mathrm{~mm}$ in thickness. These thick plane samples were treated similarly to the thin filmy samples mentioned above. The samples were first annealed in vacuum at $200^{\circ} \mathrm{C}$ for 20 days, followed by a doping process, which was terminated by rinsing with water and aged in vacuum. Finally, the samples were exposed to a saturated $\mathrm{K}_{2} \mathrm{SO}_{4}$ aqueous solution in a desiccator for more than 40 days at room temperature, in which air was stirred by a fan (swelling process). During the swelling process, we made sure the samples did not come into contact with $\mathrm{K}_{2} \mathrm{SO}_{4}$ (aqueous), which was reminded to maintain an equilibrium of $\mathrm{RH}$, which was held at $\sim 97 \%$ at room temperature. ${ }^{14}$

\section{X-ray diffraction}

WAXD patterns for all the samples were observed using a flat camera to obtain measurements through imaging plates, and monochromatized $\mathrm{MoK} \alpha$ $(\lambda=0.0711 \mathrm{~nm})$ beam was used for the incident generated by an X-ray rota unit (RIGAKU, Tokyo, Japan, RU-200, $40 \mathrm{kV} \times 50 \mathrm{~mA}$, exposed for $20 \mathrm{~min}$ ). After swelling in the desiccator, the thick plane samples were taken out, and some pieces cut from them were placed on the WAXD stage and investigated with no enclosure at room temperature; no treatment to maintain constant humidity was performed during WAXD investigation of the plane samples. In contrast, WAXD measurements of the thin filmy samples were performed within the enclosing vessel, which could maintain a constant humidity level. Intensity profiles were calculated from the imaging plate images; intensities were measured along the diameter of each image and were corrected with Lorentz factors.
Table 1 Increase in mass $(\Delta m, \%)$ for samples on each process

\begin{tabular}{lccc}
\hline Sample & $\begin{array}{c}\text { After } \\
\text { doping }\end{array}$ & $\begin{array}{c}\text { Aged in } \\
\text { vacuum }\end{array}$ & $\begin{array}{c}\text { After } \\
\text { swelling }\end{array}$ \\
\hline A (plane sample by nylon 6 pellets) & 135 & 84 & 89 \\
B (filmy sample by Rayfan NO) & 214 & 127 & - \\
\hline
\end{tabular}

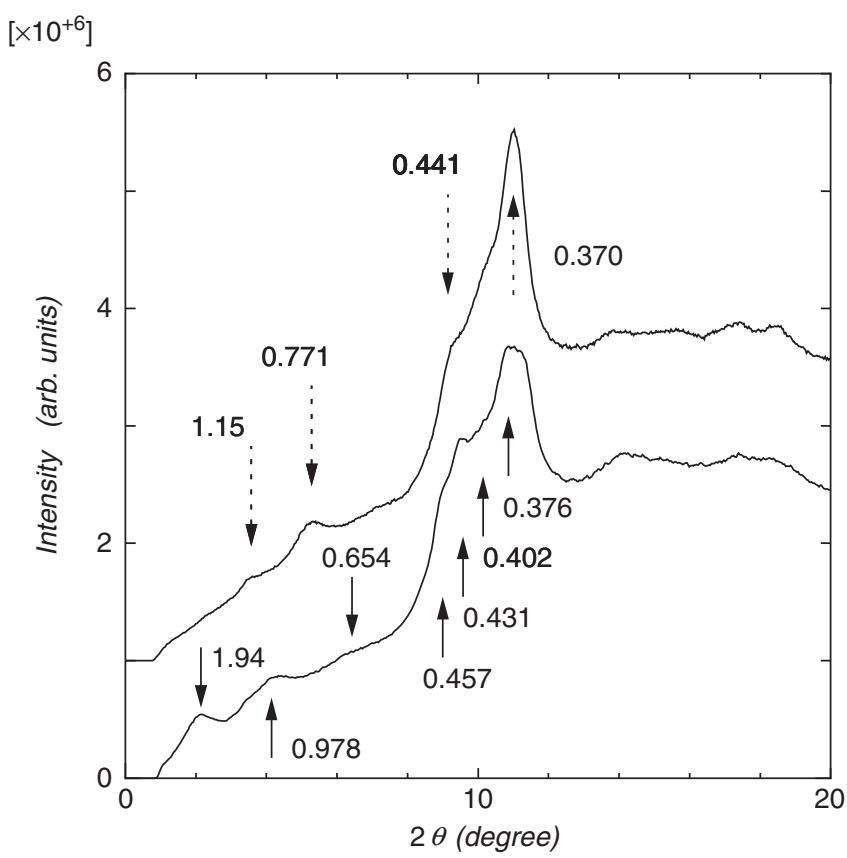

Figure 1 Wide-angle $\mathrm{X}$-ray diffraction profiles for 'sample A' aged in a desiccator immediately after removing the samples from the desiccator (lower line) and aged for $12 \mathrm{~h}$ in atmosphere (upper line, vertically shifted with $1 \times 10^{6}$ counts). Values in profiles indicate spacing $(\mathrm{nm})$; solid arrows in the lower profile indicate diffraction peaks for a fresh sample and broken arrows in the upper profile indicate ones for an aged sample.

\section{RESULTS}

Table 1 shows some cases of $\Delta m$ for the plane filmy samples through each process; $\Delta m$ values indicate increases with respect to the original undoped nylon 6 and are guides to estimate adsorption and desorption. Immediately after iodine doping, the $\Delta m$ value was $\sim 100-200 \%$ adsorption, though aging at room temperature could not induce the full release of adsorbed elements from nylon 6 . The fact that the $\Delta m$ values could not be lower than $70-80 \%$ by aging in vacuum is an indication that the dopants coordinated within the nylon 6 matrix were fixed. Aging in atmosphere or in vacuum could not produce $\Delta m$ values $<50 \%$, and some volatile components were lost during the aging process after iodine doping.

When doped iodine was not entirely released from the nylon 6 matrix, swelling modified the coordinated structure. Figure 1 is the WAXD profile of a thick plane sample aged in the desiccator at a $\mathrm{RH}$ of $\sim 97 \%$; the profile represents aging (a) immediately after taking the sample out from the humid desiccator and (b) after aging for $12 \mathrm{~h}$ under a dry atmosphere. Although the iodine-doped nylon 6 complex ordinarily shows $1.56-\mathrm{nm}$ spacing, which is attributed to the most stable intercalated structure of the complex, the swollen sample showed a different spacing. ${ }^{3,8}$ For some swollen thick plane samples, a different diffraction spacing of $1.95 \mathrm{~nm}$ was observed in their WAXD profiles immediately after swelling. However, the results from other 
$\left[\times 10^{+6}\right]$

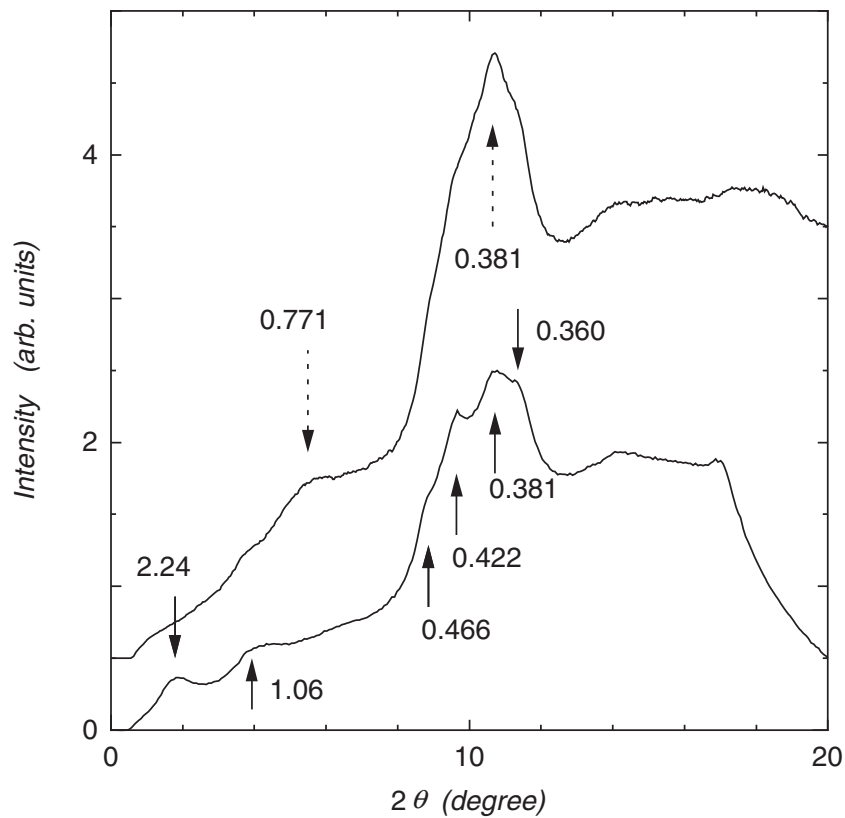

Figure 2 Wide-angle $\mathrm{X}$-ray diffraction profiles for a sample treated similarly to 'sample A' aged in a desiccator immediately after removing the sample from the desiccator (lower line) and aged for $12 \mathrm{~h}$ in atmosphere (upper line, vertically shifted with $5 \times 10^{5}$ counts). Arrow denotations are the same as those described in Figure 1.

plane samples that were similarly aged in the desiccator at constant humidity showed longer spacings $(2.1-2.2 \mathrm{~nm}$; Figure 2$)$. In addition, such new structures induced by swelling gradually vanished with aging outside the desiccator (Figures 1 and 2).

In contrast, though the $1.56-\mathrm{nm}$ spacing, which is ordinarily dominant in the complex, was hardly observed in the swollen samples, it was observed in both samples aged for $12 \mathrm{~h}$ after removing them from the moist environment of the desiccator; the 1.56-nm spacing that was lost in the swollen samples was revived through drying. In addition, in the diffraction patterns of the samples, the orientation of the intercalated structures was also observed to be similar to that observed in previous studies; however, the original plane sample of nylon 6 was not oriented. ${ }^{3,8}$

The changes in the WAXD profiles of the thin filmy samples, which were maintained at constant humidity inside the vessel, are indicated in Figure 3; the data indicate that the $\mathrm{RH}$ and temperature in the vessel were held at $65-80 \%$ and $25-30{ }^{\circ} \mathrm{C}$, respectively. However, the highhumidity level $(\mathrm{RH} \sim 97 \%)$ in the desiccator used to swell the thick plane samples could not be achieved within the vessel used to swell the thin filmy samples. Figure 3 shows that with longer aging times in the moist environment, the 1.56- $\mathrm{nm}$ spacing vanished and the $1.95-\mathrm{nm}$ spacing, which had not been observed as the stable structure in the dried sample, appeared. Moreover, the 1.95-nm spacing observed in the filmy sample also vanished on further aging in the moist environment. Still, the filmy sample did not show spacing as long as the 2.2-nm spacing of the swollen thick plane sample.

\section{DISCUSSION}

Iodine doping using polyiodide aqueous solution introduces complex structures in many hydrophilic polymers, such as poly(vinyl alcohol) (PVA) and starch. ${ }^{15-17}$ However, in general, diffusion through crystallites is clearly distinct from that occurring through the amorphous $\left[\times 10^{+3}\right]$

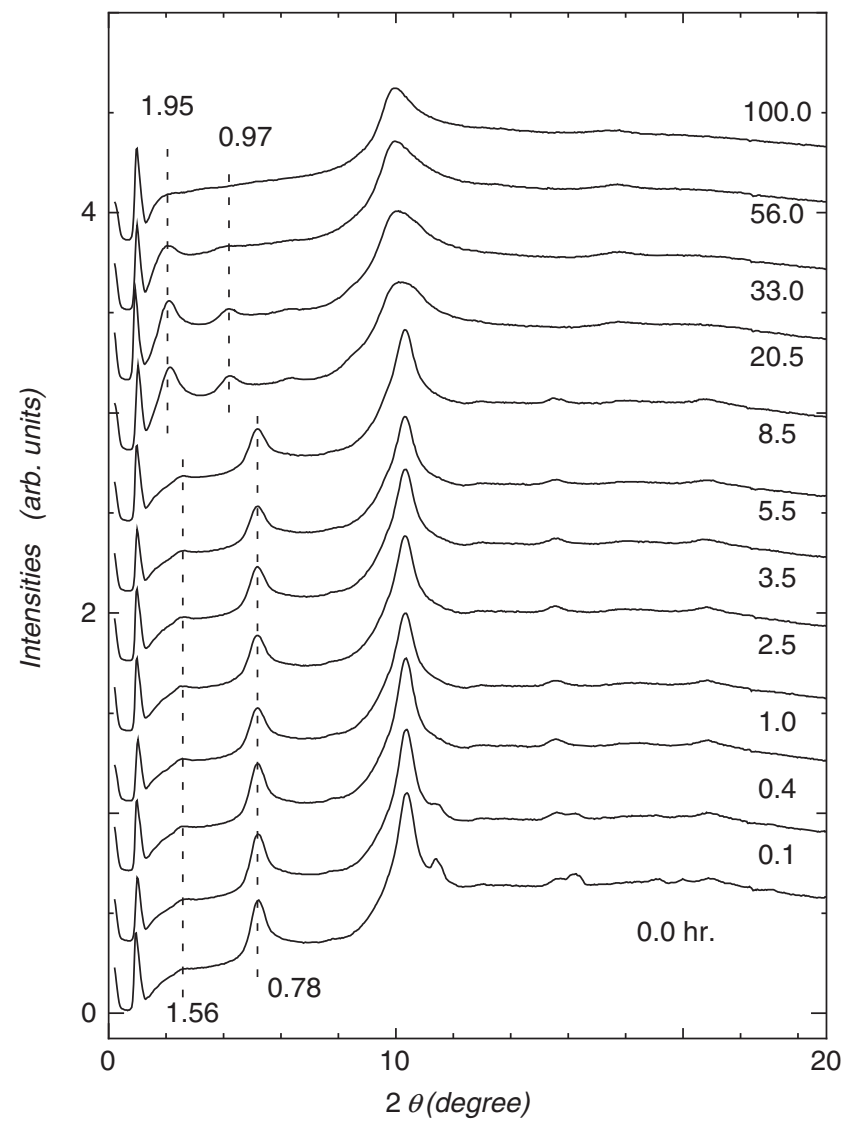

Figure 3 Wide-angle X-ray diffraction profiles for filmy 'sample B' in a closed vessel; diffraction profiles with aging time after pumping air are drawn with successive vertical shifts with $3.5 \times 10^{2}$ counts. Values in profiles followed by dotted lines indicate spacing $(\mathrm{nm})$.

regions of polymeric systems. ${ }^{18-20}$ Therefore, the number of dopants coordinated in the crystalline regions is expected to generally be less than that in the amorphous regions; moreover, even if coordination advances, ionic conduction in the crystalline regions is expected to be much less active than that in the amorphous regions. Nevertheless, during the preparation of the nylon 6/iodine complex, both coordination and diffusion of the polyiodides can immediately and easily advance in both the crystalline and the amorphous regions of nylon 6 . Furthermore, the data suggest that even the ordered structures of the complex respond to humidity.

The diversity of $\Delta m$ values between the plane and filmy samples is due to the thickness of each sample (Table 1). Iodine doping and swelling were considered to have been sufficiently achieved for both the thick plane and the thin filmy samples; swelling should promote both adsorption of vapor from humid air to the complex and ejection of the dopants from the complex. ${ }^{13}$ Cations as counter ions (in this case, potassium ion, $\mathrm{K}^{+}$) cannot be volatilized, but polyiodide ions can be converted to iodine, $\mathrm{I}_{2}$, vapor to be released outside the polymer. Unfortunately, such species complicate the results or induce low reproducibility.

Differences in the diffusion results between the 1.95-nm spacing sample (Figure 1) and the 2.2-nm spacing sample (Figure 2) were expressed even though they were treated under similar conditions in the same desiccator. The irreproducibility of the results obtained from samples tested inside the desiccator may be due to deviation in the 
diffusion behavior between the plane and filmy samples. The plane samples (0.5-1.0 $\mathrm{mm}$ in thickness) should reflect non-uniform time lag for doping, swelling or ejection. Even though iodine doping rapidly and drastically advances on the preparation of the complex, all regions of the samples cannot always release the dopants or be swollen uniformly. In addition, yet some spacings (such as the 1.15-nm spacing in Figure 1) cannot be distinctly explained. These may be second-order diffractions of the 2.2-nm structure.

The most important result obtained is that the 1.56- $\mathrm{nm}$ spacing was not always observed in the swollen plane samples, whereas the spacing could be observed to be stable in the WAXD patterns even after aging for 2 weeks or more in vacuum during the second drying process. ${ }^{12}$ It was observed that the 1.56-nm spacing, which is the most characteristic and most static for the complex at room temperature, could be obtained in the absence of moisture or water, but it vanished in a moist environment. However, the longer spacings, such as 1.95 or $2.2 \mathrm{~nm}$, were not stable through all the stages and were exhibited only in the swollen state of the complex; such spacings are attributed to meta-stable ordered states that are achieved by swelling. In the case of these plane samples, the $1.56-\mathrm{nm}$ spacing or the undoped nylon 6 $(\alpha$ - or $\gamma$-phase) samples was recovered by aging outside the enclosure. This is an indication that the 1.56-nm spacing, which was lost in the swollen samples, can be recovered by drying.

The meta-stable ordered states induced by moisture were observed as transitional stages during the advance of excess swelling. Timedependent observation of WAXD patterns in the swelling vessel indicates that the appearance and disappearance of the $1.95-\mathrm{nm}$ spacing advanced during continuous swelling. In Figure 3, the transition from the ' $1.56 \mathrm{~nm}$ structure' to the ' $1.95 \mathrm{~nm}$ structure' occurs during aging for $8.5-20.5 \mathrm{~h}$. Further swelling induces ejection of the dopants as well; after moist aging for $100 \mathrm{~h}$, no intercalated structure was observed, and diffraction of the nylon 6 (maybe $\gamma$-phase) sample was recovered. Nevertheless, compared with the thick plane samples swollen in the desiccator, the thin film samples enclosed in the vessel showed the 1.95-nm spacing only.

The reason why the 2.2-nm spacing could not be observed through the continuous swelling in the vessel cannot be answered here: it may be attributed to the lower humidity in the enclosing vessel or to the irreproducibility of the samples, as previously mentioned. It may also be attributed to the existence of some structure with 2.2-nm spacing that is at equilibrium in the plane samples aged under atmospheric conditions (Figure 2). Murthy et al. ${ }^{3}$ also reported the 2.1-nm spacing; they attributed the structure to a high density of iodine during the release process. However, the discussion of such a notion would have to consider the disturbance caused by the swelling and drying (de-swelling) process or consider the non-uniform diffusion in the hydrophilic polymer. With precise control of $\mathrm{RH}$ in the vessel, we may be able to estimate the dependence of the modified intercalation on time and humidity through future research, although it should be considered that uniform processes in the plane samples (diffusion, swelling and ejection) are complicated and that reproducibility is not easily achieved. For example, although the 1.56-nm spacing vanishes with swelling and is recovered with drying, we should not assume that the whole system can be recovered after drying-we must consider some losses by the volatilization of the dopants.

In this sense, uniform thin-film samples of nylon 6, such as Rayfan, possess advantages in achieving more uniform swelling and quick diffusion under equilibrium. However, the investigation of such thin films is also plagued by problems, such as the fact that the reorientation induced by diffusion is more effective in thin samples; for example, although re-oriented structures are observed in the plane samples (Figures 1 and 2), other re-orientation tendencies are observed in the thin, doubly oriented films. ${ }^{10}$ We will report on the modification of the orientation undoped nylon 6 induced by doping in future. ${ }^{21}$

Our results suggest that the variation and change in the ordered spacing induced by swelling can be attributed to the $\alpha-\gamma$ transition in the ordered region of the original nylon 6; the 1.56-nm and other spacings may originate in the ordered states of the complex, which originate in the crystalline regions of nylon 6 . It is expected that diffusion through the amorphous regions of nylon 6 or its complex is rapid because ionic conduction enhanced by swelling in the complex is responsive on the order of seconds or minutes. ${ }^{13}$ However, the variation in spacing may also be a function of the stability of each stage in the complex structures of nylon 6; the 1.56-nm spacing is observed in most samples and even in vacuum. Doped polyiodide ions seemed to be hardly released from the crystalline regions during spontaneous aging, although they had also drastically diffused and coordinated within minutes or seconds. ${ }^{21}$ Nevertheless, the above results suggest that slow but certain diffusion can advance in the ordered structures coordinated with iodine under high levels of humidity or under the well-swollen condition; coordination of the ions in the ordered structures of the complex is sufficiently stable, but the release of these ions or re-coordination is also possible under sufficient swelling. Thus, applied functionality in the complex can be introduced through coordination of the polyiodides or cations and activation by moisture. Because the original undoped nylon 6 is a polymer with strong hydrophilicity, it is also expected that coordination with the ions or water enhances activity in the complex. In general polymeric systems, diffusion or conduction in ordered regions, such as crystalline phases, may be negligibly lower than in amorphous regions. However, owing to the presence of iodine, diffusion in unexpected regions can be achieved.

It should be noted that such release processes during swelling induce various modifications of the intercalated structures of the complex. The 1.56-nm spacing in the nylon 6/iodine complex can be attributed to the intercalated structures in which the polyiodide ions are coordinated in all sites of the layers between nylon 6 chains. ${ }^{9}$ However, one of the modified intercalations that exhibits $1.95-\mathrm{nm}$ spacing is also observed by treatment with KI aqueous solution: the structure is regarded as a 'partially occupied intercalation', which is

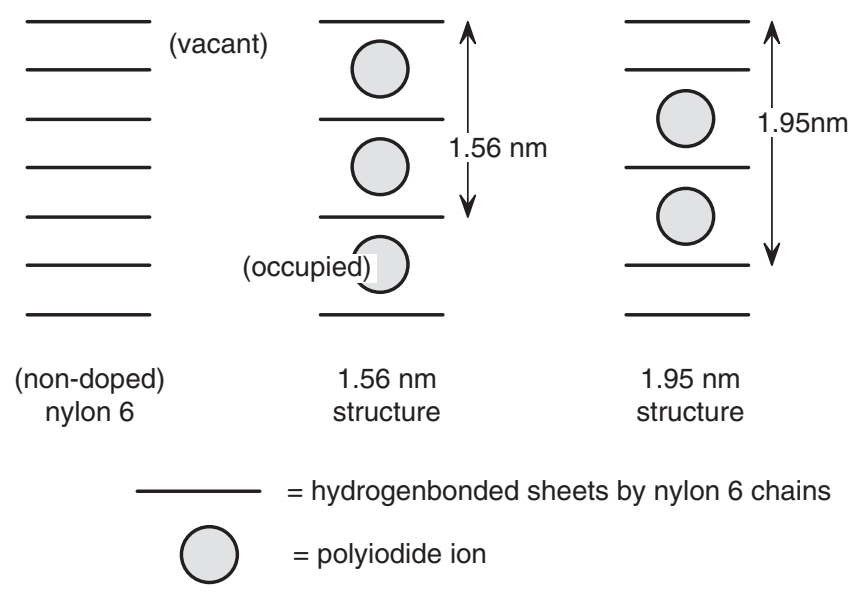

Figure 4 Scheme of intercalated structure in iodine-doped nylon 6 crystallite (parallel view along 'hydrogen-bonded sheets'); generally observed structure ( $1.56 \mathrm{~nm}$ spacing) is regarded as a fully occupied intercalation, whereas partial occupation by iodine induces the modified 1.95-nm structure due to the ejection effect by the mono-iodide ion or moisture. 9,11 
caused by the 'anti-doping' effect of the KI solution or the ejection of polyiodide ions. ${ }^{11}$ Therefore, swelling by moisture is also regarded as a procedure that can be used to induce the ejection of doped polyiodides from the ordered states. Thus, we can conclude that swelling has a similar effect as ejection by the KI solution, although the structure produced by the KI solution cannot yet be precisely identified.

If the ejection effect induced by mono-iodide ion $\left(\mathrm{I}^{-}\right)$or moisture can produce a modified intercalated structure by polyiodide ion activity, then the 'partially occupied intercalation' can be suggested as arising from the 1.95- $\mathrm{nm}$ spacing and the 'fully occupied intercalation' can be regarded as producing the $1.56 \mathrm{~nm}$-spacing due to the symmetry of the crystalline regions and the density of intercalated iodine. Figure 4 shows an intercalated structure with iodine between 'hydrogen-bonded sheets' formed by nylon 6 (anti-parallel) chains, which was suggested in previous publications. ${ }^{9} 11$ However, a model for the structure from which the 2.2-nm spacing originated cannot be presently suggested. This spacing was not effectively reproduced and was paradoxically observed in both the dilution (ejection of iodine) and the concentration processes: it may be due to some structure formed during the release process, as was reported by Murthy et al., although similar diffraction and anisotropy were indicated during the initial diffusion of iodine. ${ }^{3,22}$ Thus, our present data for the structure producing the 2.2-nm spacing in swollen iodine-doped nylon 6 cannot be concluded to originate on dilution or concentration of iodine.

Although the 2.2-nm-spaced structure, which was observed in some plane samples aged in the humid desiccators, has not been conclusively identified, we do note that the complex can introduce distinct variations in intercalation and that such intercalated structures can be discretely modified by the release process as controlled by the moisture level or the KI solution at room temperature even after the samples' fabrication.

\section{CONCLUSION}

Aging in a moist environment induces the modification of intercalated structures of nylon 6/iodine complexes. Longer spacings than the one produced by the stable structure, that is, 1.95 or $2.2 \mathrm{~nm}$, which appear as meta-stable states in the swollen complex, suggest that the intercalated structures in the ordered states respond to swelling of the complex and that coordinated polyiodide ions can be re-arranged in the ordered region after doping. These structures are meta-stable in the swollen samples compared with the stable 1.56-nm intercalation, which is often observed and even recovered in the dry sample.

\section{ACKNOWLEDGEMENTS}

We thank Professor Miyaji of Kyoto University for excellent advice and the colleagues in his laboratory for use of WAXD instruments. We thank Toray
Plastic Films for the kind supply of Rayfan NO films. Some of the results were obtained using an imaging plate reader and an analyzing system that were purchased for a project at RIKEN, 'The Development and Application of Neutron Optics (NOP)', which was supported by Special Coordination Funds for promoting the Ministry of Education and Science of the Japanese Government.

1 Arimoto, H. $\alpha-\gamma$ Transition of Nylon 6. J. Polym. Sci. Part A 2, 2283-2295 (1964).

2 Abu-Isa, I. $\alpha-\gamma$ Transition in nylon 6. J. Polym. Sci. Part A-1 9, 199-216 (1971).

3 Murthy, N. S., Szollosi, A. B. \& Sibilia, J. P. Structure of iodide ion arrays in iodinated nylon 6 and the chain orientation induced by iodine in nylon 6 films. J. Polym. Sci. Polym. Phys. Ed. 23, 2369-2376 (1985).

4 Burzynski, R., Prasad, P. N. \& Murthy, N. S. Structure of the iodine columns in iodinated nylon-6. J. Polym. Sci. Polym. Phys. Ed. 24, 133-141 (1986).

5 Murthy, N. S. Structure of iodide ions in iodinated nylon 6 and the evolution of hydrogen bonds between parallel chains in nylon 6. Macromolecules 20, 309-316 (1987).

6 Murthy, N. S., Hatfield, G. R. \& Glans, J. H. X-ray diffraction and nuclear magnetic resonance studies of nylon $6 / I_{2} / \mathrm{KI}$ complexes and their transformation into the $\gamma$ crystalline phase. Macromolecules 23, 1342-1346 (1990).

7 Murthy, N. S. \& Khanna, Y. P. Transformation between polyiodide structures in a nylon 6 matrix. Chem. Mater. 5, 672-677 (1993).

8 Kawaguchi, A. Structure of iodine-nylon 6 complex: 1 . The investigation of the lattice constants and hydrostatic compression of the complex crystal. Polymer 33, 3981-3984 (1992).

9 Kawaguchi, A. Structure of iodine-nylon 6 complex: 2. Arrangement of polyiodides in the complex. Polymer 35, 2665-2671 (1994).

10 Kawaguchi, A. Structure of iodine-nylon 6 complex: 3. Modification of double orientation of the complex with doping. Polymer 35, 3797-3798 (1994).

11 Kawaguchi, A. Structure of iodine-nylon 6 complex: 4. Irregular stacking of intercalated iodine in the complex. Polymer 37, 4877-4880 (1996).

12 Kawaguchi, A. Response of iodine/nylon 6 complex to humidity: 2. activation by humidity and cations. Sens. Actuators B 63, 10-17 (2000).

13 Kawaguchi, A. Response of iodine/nylon 6 complex to humidity: 3. Discussion based on increase in mass. Sens. Actuators B 73, 174-178 (2001).

14 Inamatsu, T. in Kobunshi To Mizu (in Japanese) (ed. Soc. Polym. Sci. Jpn.) Ch.10, 203-224 (Kyoritsu Shuppan, Tokyo, 1995).

15 Rundle, R. E. \& Baldwin, R. R. The configuration of starch and the starch-iodine complex. I the dichroism of flow of starch-iodine solutions. J. Am. Chem. Soc. 65, 554-558 (1943).

16 Choi, Y. S., Oishi, Y. \& Miyasaka, K. Structure of poly(vinyl alcohol)-iodine complex formed in the amorphous phase of poly(vinyl alcohol) films. Polym. J. 22, 601-608 (1990).

17 Tashiro, K., Minagawa, Y., Kobayashi, M., Morita, S., Kawai, T. \& Yoshino, K. Crystal structural change of poly(3-Alkylthiophene) induced by iodine doping as revealed by X-ray diffraction and infrared/raman spectroscopic measurements. Jpn. J. Appl. Phys. 33, L1023-L1026 (1994).

18 Vieth, W. R. Diffusion In and Through Polymers Ch.4 73-110 (Hanser Publishers, Munich, 1991).

$19 \mathrm{Kim}, \mathrm{H}$. \& Harget, P. J. Glass transition temperature of nylon 6 -inorganic salt mixtures. J. Appl. Phys. 50, 6072-6076 (1979)

20 Burford, R. P. \& Harrauer, E. The diffusion of salt solutions into nylon-6. Polymer 24, 1001-1007 (1983).

21 Kawaguchi, A., Tsurutani, N., Fukao, K. \& Miyaji, H. Investigation of re-orientation for nylon on iodine-doping. SPring-8 User Exp. Rep. 5, 354 (2000).

22 Kawaguchi, A., Tsurutani, N., Fukao, K., Koyama, A., Tahara, D., Yamao, H., Toda, A., Miyamoto, Y. \& Miyaji, H. Investigation of structure for nylon 6 and other hydrophilic polymers on iodine-doping. SPring-8 User Exp. Rep. 7, 227 (2001). 